Año 13.

Revista de Investigación

Núm. 33

Académica sin Frontera

https://revistainvestigacionacademicasinfrontera.unison.mx/index.php/RDIASF

Recibido el 30 de abril de 2020. Dictaminado mediante arbitraje favorablemente 22 de septiembre de 2020.

\title{
Idea a defender: una concepción teórico-metodológica para su tratamiento en las tesis de maestría y doctorado.
}

Autor:

Dr.C. Ricardo Alcibiades Carmona-Hernández, Filial Universitaria Guáimaro. Universidad de Camagüey. https://orcid.org/0000-0001-9825-0697

Coautores:

M.Sc. Andrés Anesio Fernández-Villalobos, Filial Universitaria Guáimaro. Universidad de Camagüey. //orcid.org/0000-0001-8819-3502

M.Sc. Félix Alberto Laguna Orama, Filial Universitaria Guáimaro. Universidad de Camagüey. https://orcid.org/0000-0002-1351-7012

Dirección: Calle Libertad nº 16 entre Iris y Unión. Guáimaro. Provincia de Camagüey. Cuba.

Código postal: 72600

Teléfono: 32811248.

Correo electrónico: andres.ffernandez@reduc.edu.cu

Institución: Universidad de Camagüey.

\section{Resumen}

La utilización de idea a defender en las tesis de maestría y doctorados en ciencias defendidas en Cuba se ha incrementado, lo que refleja la cultura alcanzada al identificar aquellos componentes que constituyen el diseño teórico y la esencia del problema científico investigado. Sin embargo, el crecimiento cuantitativo de estas tesis como resultado de la política de incrementar la categoría científica en los diferentes claustros universitarios y la insuficiente precisión metodológica de este componente en la literatura 
Año 13.

Revista de Investigación

Núm. 33

Académica sin Frontera

https://revistainvestigacionacademicasinfrontera.unison.mx/index.php/RDIASF

Recibido el 30 de abril de 2020. Dictaminado mediante arbitraje favorablemente 22 de septiembre de 2020.

ha provocado en ocasiones carencias en la estructuración de ideas a defender que presentan los autores de tesis, lo cual afecta la calidad y el impacto científico posterior de estas investigaciones. En el presente trabajo se exponen algunas valoraciones al respecto que pueden contribuir a solucionar esta deficiencia.

Palabras clave: idea; defender; investigación; diseño teórico.

\section{Idea to defend: A theoretical-methodological conception for its treatment on Master and Doctorate thesis}

\section{Abstract}

The use of the idea to defend in the masters and doctorate theses in sciences defended in Cuba has increased, which reflects the culture achieved by identifying those components that constitute the theoretical design and the essence of the investigated scientific problem. However, the quantitative growth of these theses as a result of the policy of increasing the scientific category in the different university cloisters and the insufficient methodological precision of this component in the literature has sometimes caused deficiencies in the structuring of ideas to be defended presented by the thesis authors, which affects the quality and subsequent scientific impact of these investigations. In the present work, some assessments are presented in this regard that may contribute to solving this deficiency.

Keywords: idea; defend; investigation; theoretical design.

\section{Introducción}

La formulación de hipótesis ha estado relacionada al progreso histórico de la metodología de la investigación, ya que los primeros desarrollos metodológicos se hicieron dentro del 
Año 13.

Núm. 33
Revista de Investigación

Académica sin Frontera

ISSN: 2007-8870

https://revistainvestigacionacademicasinfrontera.unison.mx/index.php/RDIASF

Recibido el 30 de abril de 2020. Dictaminado mediante arbitraje favorablemente 22 de septiembre de 2020.

positivismo, para los cuales la investigación tiene como finalidad verificar las hipótesis que se derivan de alguna teoría, y su método, requería su verificación.

Para los positivistas toda investigación debía expresar una relación de causaefecto, y por lo tanto las hipótesis en su definición estricta, y como respuesta tentativa a la pregunta, también implicaban causa-efecto. Además, ellos no hablaban de tipos de investigación, sino de diseños, porque según su criterio solo la investigación confirmatoria era "valida" y "científica" (Hurtado, 2013: 1).

Como el modelo positivista predominó muchos años en el contexto de la investigación, quedó profundamente grabada la idea de que una investigación necesariamente tiene que tener una hipótesis, porque si no, no alcanza la categoría de "científica". Por esa razón, a medida que se fueron reconociendo y reconceptualizando otros tipos de investigación, los investigadores se vieron forzados a utilizar a toda costa la hipótesis para justificar el carácter científico de la investigación, incluso en aquellos casos en que las hipótesis no tienen razón de existir ni aportan al proceso metodológico.

\section{Justificación}

En la enseñanza superior en Cuba:

$\checkmark$ En un número significativo de trabajos científicos se usa la idea a defender como variante de hipótesis, en la cual no se comprende su estructura para ser utilizada como modelo por otros que incursionan en su utilización.

$\checkmark$ La causa fundamental de esta situación, es la falta de definición de una concepción metodológica de la idea a defender como tipo de hipótesis con una sola variable. 
Año 13.

Núm. 33
Revista de Investigación

Académica sin Frontera

ISSN: 2007-8870

https://revistainvestigacionacademicasinfrontera.unison.mx/index.php/RDIASF

Recibido el 30 de abril de 2020. Dictaminado mediante arbitraje favorablemente 22 de septiembre de 2020.

\section{Planteamiento del problema}

De lo expuesto anteriormente se precisa como problema científico, la concepción metodológica de la idea a defender, como objeto, el proceso de investigación científica y como campo, la teoría y metodología para el tratamiento de la idea a defender.

Y como objetivo, elaborar una concepción teórico-metodológica para el tratamiento de la idea a defender para su uso en las tesis de maestría y doctorado.

En el desarrollo del trabajo se cumplimentaron los siguientes objetivos específicos:

1. Sistematización de los fundamentos teóricos en los que se sustenta el uso de la idea a defender.

2. Caracterización del diagnóstico que presenta la educación superior en cuanto al uso de la idea a defender.

3. Elaboración de una concepción metodológica de la idea a defender.

4. Valoración de la efectividad de la concepción metodológica propuesta de la idea a defender.

\section{Antecedentes}

En el proceso de realización del trabajo se trabajó con una amplia bibliografía que abarcan diferentes artículos y tesis que tratan el tema de las hipótesis y en especial de la variante idea a defender como son: la guía metodológica para la realización de tesis de grado de la Universidad Mayor de San Andrés, en Bolivia; La formulación de hipótesis ha estado relacionada al progreso histórico de la metodología de la investigación, ya que los primeros desarrollos metodológicos se hicieron dentro del positivismo, para los cuales la investigación tiene como finalidad verificar las hipótesis que se derivan de alguna teoría, y su método, requería su verificación. 
Revista de Investigación

Año 13.

Académica sin Frontera

Núm. 33

ISSN: 2007-8870

https://revistainvestigacionacademicasinfrontera.unison.mx/index.php/RDIASF

Recibido el 30 de abril de 2020. Dictaminado mediante arbitraje favorablemente 22 de septiembre de 2020.

Para los positivistas toda investigación debía expresar una relación de causaefecto, y por lo tanto las hipótesis en su definición estricta, y como respuesta tentativa a la pregunta, también implicaban causa-efecto. Además, ellos no hablaban de tipos de investigación, sino de diseños, porque según su criterio solo la investigación confirmatoria era "valida" y "científica" (Hurtado, 2013: 1).

Como el modelo positivista predominó muchos años en el contexto de la investigación, quedó profundamente grabada la idea de que una investigación necesariamente tiene que tener una hipótesis, porque si no, no alcanza la categoría de "científica". Por esa razón, a medida que se fueron reconociendo y reconceptualizando otros tipos de investigación, los investigadores se vieron forzados a utilizar a toda costa la hipótesis para justificar el carácter científico de la investigación, incluso en aquellos casos en que las hipótesis no tienen razón de existir ni aportan al proceso metodológico.

La palabra hipótesis, está conformada etimológicamente por las expresiones /hypo/, del griego, que indica subordinación y significa situar bajo algo, lo que apoya algo, y /thesis/, también del griego, que significa proposición. Alude a una proposición subordinada, en este caso, la hipótesis está subordinada a la teoría de la cual fue derivada.

Al revisar la definición de hipótesis de autores como Kerlinger (1981), es una expresión conjetural de la relación que existe entre dos o más variables, que además debe ser verificable a través de la experiencia. Para Mc Guigan (1990) la hipótesis es una solución tentativa al problema de investigación, que se formula como una proposición susceptible de ser sometida a prueba, y que expresa una posible relación entre una y otra variable.

Según Álvarez (1995: 20), la hipótesis es una suposición científicamente fundamentada y novedosa acerca de los componentes y sus relaciones que conforman 
Revista de Investigación

Año 13.

Académica sin Frontera

Núm. 33

ISSN: 2007-8870

https://revistainvestigacionacademicasinfrontera.unison.mx/index.php/RDIASF

Recibido el 30 de abril de 2020. Dictaminado mediante arbitraje favorablemente 22 de septiembre de 2020.

el objeto de estudio y mediante la cual se le da solución al problema de investigación. En el proceso de formulación de la hipótesis se hace necesario una abstracción para precisar lo que el investigador entiende como lo más esencial del proceso estudiado para conocer el objeto, lograr el objetivo y resolver el problema.

Este autor considera que la hipótesis como predicción, suposición y proposición, deja sentada las posibles relaciones causales, genéticas, estructurales-funcionales, dialécticas, cuyo desconocimiento generaron el problema y donde se establecen las propiedades, las variables, así como las relaciones existentes entre ellas y donde se prevén los métodos a utilizar en la investigación. Esto hace de la hipótesis el elemento o categoría rectora del Proceso de Investigación Científica (1995: 21).

En este sentido, Viciedo (1996) expone que la hipótesis es una proposición que expresa un juicio, una conjetura, una suposición que afirma algo y que generalmente enlaza por lo menos dos elementos o aspectos que se denominan variables; es una posible respuesta al problema científico y es el enlace entre la teoría y el trabajo empírico, además, se apoya en una teoría científica. La relación entre variables permite explicar y a veces predecir, los fenómenos que se estudian.

Hernández, Fernández y Baptista (2006: 122) expresan que la hipótesis nos indica lo que estamos buscando o tratando de probar y pueden definirse como explicaciones tentativas del fenómeno investigado a manera de proposiciones, acerca de las relaciones entre dos o más variables y se apoyan en conocimientos organizados y sistematizados. Por su parte G. Pérez y colaboradores (1996: 21) expresan que la hipótesis es una forma especial del conocimiento que presenta sus particularidades: nos anuncia las posibles leyes y características esenciales que esperamos encontrar en los fenómenos estudiados, por lo que representa un conocimiento probable sobre la realidad. 
Revista de Investigación

Año 13.

Académica sin Frontera

Núm. 33

ISSN: 2007-8870

https://revistainvestigacionacademicasinfrontera.unison.mx/index.php/RDIASF

Recibido el 30 de abril de 2020. Dictaminado mediante arbitraje favorablemente 22 de septiembre de 2020.

El criterio de (Hernández, Fernández y Baptista, 1997: 127-135), es que estas proposiciones se apoyan en conocimientos organizados, además pueden clasificarse en tres grupos: las correlacionadas, las de diferencia de grupos y las causales. Las hipótesis correlacionadas establecen relación entre dos o más variables, llegando en algunos casos a explicar esa relación; las de diferencias de grupos establecen desigualdades entre dos o más grupos respecto a una variable seleccionada, llegando en algunos casos a definir el sentido en que opera dicha diferencia y las formulaciones causales intentan explicar las causas y efectos de la relación entre las variables o de las diferencias entre los grupos.

La hipótesis, es un juicio de carácter especial, que puede manifestarse como una afirmación o una negación de algo, por ello en relación a su esencia es un juicio científico, técnico o ideológico, que lleva implícita un valor, un significado, una solución específica al problema. El contenido de solución que le suministramos al problema de investigación es la variable y en la hipótesis intervienen:

$\checkmark$ Variable independiente (VI): es la causa supuesta por el investigador, que produce 0 actúa en la variable dependiente,

$\checkmark$ Variable dependiente (VD): es el efecto originado o producido por la variable independiente, supuesto por el investigador.

$\checkmark$ Variable interviniente $o$ ajena: es aquella que no es causa ni efecto, pero necesita ser controlada para evitar modificaciones en las condiciones del problema investigado.

En algunos contextos también se alude a las hipótesis como supuestos o ideas que pueden ser defendidas o argumentadas de forma lógica, con lo cual se justifica que puedan formularse durante una descripción o una comparación, sin embargo, en lo que respecta a argumentar o defender un supuesto, puede decirse que no todo supuesto es una hipótesis, pues tendría que cumplir ciertas condiciones. 
Año 13.

Académica sin Frontera

Núm. 33

ISSN: 2007-8870

https://revistainvestigacionacademicasinfrontera.unison.mx/index.php/RDIASF

Recibido el 30 de abril de 2020. Dictaminado mediante arbitraje favorablemente 22 de septiembre de 2020.

Es cierto que es posible argumentar un supuesto, por ejemplo, en una investigación analítica, cuando se supone que algunos contenidos se corresponden con alguna ideología, o con ciertas interpretaciones, pero ese supuesto no es en sentido estricto una hipótesis porque no implica relaciones de causalidad o relaciones explicativas.

De acuerdo con Viciedo (1996), algunos autores señalan que, en el caso de las investigaciones experimentales, las hipótesis son imprescindibles para orientar el proceso de investigación, también plantea que, hay aproximaciones que pueden ser sustituidas por preguntas o tareas científicas, según el tipo de investigación.

Viciedo (1996) es de la opinión que, si se hace referencia a una idea a defender como sinónimo de hipótesis, es importante aclarar que no es lo mismo una idea a defender que una hipótesis a verificar. Una idea se puede defender mediante una argumentación lógica, pero en ese caso no se estará haciendo uso de la investigación sino del razonamiento como vía para generar conocimiento. En síntesis, las hipótesis se formulan en investigaciones donde se trabajan relaciones causa efecto que se van a verificar, específicamente en la confirmatoria y en la evaluativa.

En las definiciones antes mencionadas, en su concepción original, las hipótesis implican relaciones, explicaciones, desigualdades y formulaciones causales, por lo tanto, sólo se pueden formular en las investigaciones donde las variables participantes establecen este tipo de conexión, o sea, donde su objetivo es precisamente verificar la relación entre las variables o en las que su objetivo es evaluar si la acción que se ejecutó originó el efecto deseado.

En la literatura científica la hipótesis tiene una teoría suficientemente desarrollada, tratada por diferentes autores en múltiples libros y artículos, sin embargo, la idea a defender, es muy poco definida en la literatura y cuando aparece el término los 
Revista de Investigación

Año 13.

Académica sin Frontera

Núm. 33

ISSN: 2007-8870

https://revistainvestigacionacademicasinfrontera.unison.mx/index.php/RDIASF

Recibido el 30 de abril de 2020. Dictaminado mediante arbitraje favorablemente 22 de septiembre de 2020.

argumentos son débiles, no queda claro qué es, cuándo se usa y cuál es su estructura, en ocasiones se enuncian como relación causal y en otras no se percibe variables, ni siguen ningún patrón en su estructura, por ello, para tratar de comprender el término idea a defender, es válido penetrar en su origen, significado y en las propias consideraciones explícitas e implícitas.

La etimología del concepto "idea” proviene del griego ठźa, de eidon, deduciéndose como primero y más obvio de los actos del entendimiento, que se limita al simple conocimiento de algo; lo que se ve, forma, imagen que existe o se halla en la mente; imagen o representación que del objeto percibido queda en la mente; conocimiento puro, racional, debido a las naturales condiciones de nuestro entendimiento. La capacidad humana de contemplar ideas está asociada a la facultad de razonamiento, autorreflexión, creatividad y la habilidad de adquirir y aplicar el intelecto (Microsoft Encarta 2008). Desde la lingüística una idea es el contenido cognitivo o comprensión intuitiva que tiene un individuo de un tema, asunto o estado de hechos.

En la literatura filosófica, al definir idea no existe una concepción única, se plantea que es un término que designa sentido, significación, esencia y se halla estrechamente vinculado a las categorías del pensar y el ser. En el materialismo dialéctico, la idea se considera como reflejo de la realidad objetiva, también se entiende como una de las formas o procedimientos de la cognición, como forma o procedimiento cuyo sentido estriba en formular un principio teórico generalizado que explique la esencia, la ley de los fenómenos (Rosental y Ludin, 1981: 229).

Según Fernández (2001) en la mayoría de los tratados, se puede analizar la idea bajo cuatro puntos de vista:

Lógico: la idea es equiparable a un concepto, que tiene un significado.

Ontológico: la idea es equiparable a algo material que existe en el mundo real. 
Revista de Investigación

Año 13.

Académica sin Frontera

Núm. 33

ISSN: 2007-8870

https://revistainvestigacionacademicasinfrontera.unison.mx/index.php/RDIASF

Recibido el 30 de abril de 2020. Dictaminado mediante arbitraje favorablemente 22 de septiembre de 2020.

Trascendental: la idea como una posibilidad del conocimiento, es algo con lo que también se quiere dar a expresar algo para tener más conocimiento del que se tiene acerca de lo que se esta tratando o estudiando.

Psicológico: la idea es equiparable a una representación mental subjetiva.

La capacidad humana de contemplar ideas está asociada a la capacidad de raciocinio, autorreflexión, creatividad y habilidad de adquirir y aplicar el intelecto. Las ideas dan lugar a los conceptos, los cuales son la base de cualquier tipo de conocimiento, tanto científico como filosófico.

$Y$ el término defender, se define como sostener una idea, un juicio; mantener una afirmación; apoyar una idea o una teoría; interceder o hablar favorablemente de una cosa; conseguir en una determinada actividad un resultado; guardar o proteger (Larousse, 2007).

Por tanto, la unión de los términos idea y defender origina en metodología de la investigación la categoría idea a defender que pudiera ser entendida como el sostenimiento de un conocimiento puro, racional, debido al entendimiento humano de hechos, de datos extraídos de la práctica, de razonamientos construidos a partir de la teoría y la elaboración intelectual del investigador_que explica la esencia del fenómeno, lo cual constituye la posible solución del problema que se investiga. Antes de continuar es acertado expresar que la idea a defender es utilizada, principalmente, en investigaciones pedagógicas.

Para Córdova, en una investigación cualitativa o donde predomine el enfoque cualitativo no se recomienda una hipótesis, por lo menos en su forma clásica, en este caso se utiliza la idea a defender. Dicha idea no necesita ajustarse a variables, ella recoge los elementos básicos esenciales a fundamentar, considera que es una forma de hipótesis cualitativa y puede consignarse como tal, es menos encubierta que las 
Año 13.

Académica sin Frontera

Núm. 33

ISSN: 2007-8870

https://revistainvestigacionacademicasinfrontera.unison.mx/index.php/RDIASF

Recibido el 30 de abril de 2020. Dictaminado mediante arbitraje favorablemente 22 de septiembre de 2020.

"preguntas científicas", no niega ser sintética y con el cambio de nombre elude las grandes pretensiones y compromisos.

Según López (2007) la idea a defender pretende escapar a la supuesta obligatoriedad de un profundo proceso de constatación de la hipótesis y, esencialmente, a la aplicación de una rigurosa operacionalización y su correspondiente tratamiento estadístico-matemático.

De acuerdo con Cerezal y Fiallo (2002: 34), se ha difundido mucho la utilización de ideas a defender, en sustitución de hipótesis o preguntas científicas, se basa en un supuesto teórico que plantea el investigador y que trata de justificar en el desarrollo de su investigación. Dicen, que se toca las manos con la hipótesis, la diferencia está en que se utiliza no sólo en investigaciones de corte experimental, sino también, en investigaciones descriptivas.

Los autores antes mencionados con relación a la idea a defender son del criterio que se ha convertido en una variante entre la hipótesis y las preguntas científicas, pues indudablemente tiene rasgos de la primera, pero consideran que no cumple con rigurosidad los componentes estructurales que debe poseer una hipótesis.

Según Lanuez, Martínez y Pérez (2004: 66) la idea a defender es un juicio hipotético, pues plantea la solución anticipada del problema, de ahí que se considere que es una hipótesis sin los requisitos de ésta, además de que no necesita la contrastación empírica con el rigor en el control de las variables que precisa la hipótesis.

La idea a defender tiene similitud con la hipótesis, la diferencia es que la hipótesis es utilizada para investigaciones cuantitativas que deben ser probadas matemáticamente, en cambio, la idea a defender es de carácter cualitativo, es decir, un supuesto de lo que puede pasar con la aplicación de la investigación (Comisión académica UPEC, 2011: 11). 
Revista de Investigación

Año 13.

Académica sin Frontera

Núm. 33

ISSN: 2007-8870

https://revistainvestigacionacademicasinfrontera.unison.mx/index.php/RDIASF

Recibido el 30 de abril de 2020. Dictaminado mediante arbitraje favorablemente 22 de septiembre de 2020.

En este sentido, consideramos que la idea a defender es un tipo de hipótesis, por lo que es una predicción científica, que de forma afirmativa puntualiza las cualidades, características y puntos de vista del investigador respecto a la solución del problema científico. Indica por vía sintética el nuevo conocimiento que se revela como solución al problema, sin llegar a expresar la relación dialéctica causa efecto entre las variables, pues sólo contiene la variable independiente.

Para que una predicción sea una idea a defender debe reunir las siguientes características:

$\checkmark$ Estar redactada en términos afirmativos.

$\checkmark$ Expresar una conjetura científica.

$\checkmark$ Debe estar sustentada en un aparato teórico referencial.

$\checkmark$ No puede evidenciar condicionamiento.

$\checkmark$ Es necesario que se redacte en términos de las cualidades, características o elementos teóricos en que se sustenta la solución, de manera que permita visualizar lo novedoso en la investigación, pero no el efecto, por tanto, debe contener la variable independiente, pero no la dependiente.

A partir de las deficiencias encontradas en ideas a defender enunciadas en tesis en opción a diferentes categorías científicas y en busca de un criterio que permita a los investigadores justificar y argumentar la idea formulada, proponemos la siguiente estructura de la idea a defender:

$\checkmark$ Unidades de observación: son las personas, grupos, objetos, actividades e instituciones sobre lo que versa la investigación.

$\checkmark$ Variable independiente: es la causa, el resultado científico que se revela como posible solución al problema. 
Revista de Investigación

Año 13.

Académica sin Frontera

Núm. 33

ISSN: 2007-8870

https://revistainvestigacionacademicasinfrontera.unison.mx/index.php/RDIASF

Recibido el 30 de abril de 2020. Dictaminado mediante arbitraje favorablemente 22 de septiembre de 2020.

$\checkmark$ Lo que persigue el investigador con la variable independiente en su estudio: este elemento evidencia el cambio o transformación que se lograría si se aplicara la idea a defender. Para revelar lo que se persigue en la formulación de la hipótesis, se pueden utilizar términos como orientada, dirigida, encaminada, destinada, enfocada, entre otros

Las cualidades, características o elementos teóricos de la variable independiente: este componente explica las particularidades de la variable independiente y lo que distingue su propuesta de otras. Para enunciar este componente en la hipótesis, se pueden utilizar términos como, será portadora, incluirá, contendrá, comprenderá y sus equivalentes.

$\checkmark$ Los términos lógicos: son los que relacionan las unidades de observación con la variable, con lo que persigue y con sus cualidades.

Por ello, la forma en que proponemos que se enuncia la idea a defender es:

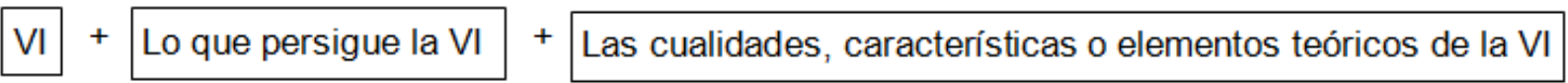

Como se puede apreciar, en la estructura de la idea a defender que proponemos, solo aparece la variable independiente, por lo que en los estudios donde se utilice esta categoría la finalidad es el descubrimiento de los elementos que caracterizan la acción propuesta como solución o son investigaciones exploratorias, descriptivas o cualitativas. Por ello, su uso no persigue la verificación de un supuesto previo y como consecuencia de ello, no se establece una relación de dependencia entre variables ni se espera un efecto o un cambio en el fenómeno observado, sino que, se pretende que el investigador evidencie lo que persigue y las cualidades, características o elementos teóricos de la variable independiente. 
Año 13.

Revista de Investigación

Núm. 33

Académica sin Frontera

ISSN: 2007-8870

https://revistainvestigacionacademicasinfrontera.unison.mx/index.php/RDIASF

Recibido el 30 de abril de 2020. Dictaminado mediante arbitraje favorablemente 22 de septiembre de 2020.

Por ejemplo:

Una estrategía de superación orientada al desarrollo de las acciones intelectuales necesarias para la formulación de problemas, será portadora de las relaciones esenciales entre la estructuración didáctica de la formulación de problemas matemáticos con texto y la fundamentación, el diseño y la ejecución de las formas de Educación Avanzada que la integran. I

Cualidades

\section{¿Cómo transformar una hipótesis causal en idea a defender?}

Para transformar una hipótesis causal en idea a defender, se enuncia la variable dependiente o efecto esperado como lo que persigue la variable independiente en el estudio y los elementos que explican las particularidades de la variable independiente en el fenómeno estudiado o dan razones de por qué el fenómeno se presenta de una manera y no de otra, se declaran como sus cualidades. 
Año 13.

Revista de Investigación

Núm. 33

Académica sin Frontera

ISSN: 2007-8870

https://revistainvestigacionacademicasinfrontera.unison.mx/index.php/RDIASF

Recibido el 30 de abril de 2020. Dictaminado mediante arbitraje favorablemente 22 de septiembre de 2020.

Por ejemplo, una hipótesis sería:

Persigue

Se puede contribuir a eliminar las insuficiencias que se manifiestan en el comportamiento profesional y social de los estudiantes universitarios en correspondencia con las exigencias de su formación integral, si se establece una Estrategia de Gestión, basada en un Modelo de Gestión de Calidad del proceso de formación del profesional, que se sustente en la contradicción dialéctica entre el compromiso y la flexibilidad expresada en el protagonismo de los estudiantes.

\section{Cualidades}

Causa

Esta hipótesis se puede transformar en la siguiente idea a defender, con la estructura que proponemos:

Esta hipótesis se puede transformar en la siguiente idea a defender, con la estructura que proponemos:

\section{Causa}

Persigue

Una Estrategia de Gestión dirigida a la eliminación de las insuficiencias que se manifiestan en el comportamiento profesional y social de los estudiantes universitarios en correspondencia con las exigencias de su formación integral comprenderá un Modelo de Gestión de Calidad del proceso de formación del profesional, que se sustente en la contradicción dialéctica entre el compromiso y la flexibilidad expresada en el protagonismo de los estudiantes. 
Revista de Investigación

Año 13.

Académica sin Frontera

Núm. 33

ISSN: 2007-8870

https://revistainvestigacionacademicasinfrontera.unison.mx/index.php/RDIASF

Recibido el 30 de abril de 2020. Dictaminado mediante arbitraje favorablemente 22 de septiembre de 2020.

\section{Diagnóstico}

Para diagnosticar el estado inicial se realizó una encuesta a 22 docentes del Centro Universitario Municipal Guáimaro que arrojó lo siguiente:

- El $81 \%$ de los docentes (18) no conocen la estructuración de ideas a defender, que trae errores en su utilización en diferentes investigaciones.

- El $72 \%$ de los encuestados (16) no utiliza la idea a defender al no poseer los conocimientos necesarios para su uso.

Los resultados y discusión de la propuesta: Para la valoración de la propuesta se acudió a un grupo de especialistas (10), integrados por docentes de larga experiencia en temas de investigación, superior en todos los casos a 15 de años, para que, mediante el método de talleres de opinión crítica y construcción colectiva, llegar a conclusiones. Los resultados de estas opiniones fueron:

- Se define con este trabajo una concepción metodológica de la idea a defender como tipo de hipótesis con una sola variable, siendo viable a partir de ello su uso en investigaciones, principalmente de corte pedagógico.

\section{Conclusiones}

La ciencia y la metodología de la investigación se encuentran en un momento de progreso en el plano científico, muchos investigadores han hecho aportes en este campo, lo que ha ocasionado una variedad metodológica en cuanto a sus conceptos, sin embargo, en relación a esta categoría científica tan importante en el proceso de investigación, en la literatura no existe claridad metodológica y como consecuencia de ello, existe incertidumbre de su uso por un grupo de investigadores. 
Año 13.

Revista de Investigación

Núm. 33

Académica sin Frontera

https://revistainvestigacionacademicasinfrontera.unison.mx/index.php/RDIASF

Recibido el 30 de abril de 2020. Dictaminado mediante arbitraje favorablemente 22 de septiembre de 2020.

De ahí que surja, como una necesidad, valorar y fundamentar los elementos que tipifican la idea a defender en el proceso investigativo. Por ello, en este estudio, desde lo teórico y lo metodológico, hemos definido esta categoría, hemos propuesto una estructura para ella y cuando se debe usar.

\section{Bibliografía}

1. Álvarez, C. (1995). Metodología de la investigación científica. Centro de Estudios de Educación Superior "Manuel F. Gran", Universidad de Oriente. Santiago de Cuba, Cuba. Obtenido de https://scholar.google.com.cu

2. Campistrous, L., \& Rizo, C. (1999). ¿Hipótesis o preguntas científicas en los trabajos de investigación? Ciudad de la Habana, Cuba. Obtenido de http://aulavirtual.uij.edu.cu

3. Cerezal, J., \& Fiallo, J. (2002). Los métodos científicos en las investigaciones pedagógicas. Ciudad de la Habana, Cuba. Obtenido de www.clubensayos.com

4. Colectivo de autores. (2010). Guía metodológica para la realización de tesis de grado de la Universidad Mayor de San Andrés, La Paz. Bolivia.

5. Comisión académica UPEC. (2011). Manual para la elaboración de tesis de grado. Tulcán, Ecuador. Obtenido de https://docplayer.es

6. Córdova, C. (Material en soporte magnético sin fechar). Consideraciones sobre la metodología de la investigación. Universidad de Holguín "Oscar Lucero Moya", Centro de estudios sobre la cultura e identidad. Holguín, Cuba.

7. Díaz Torres, R. (2016). HIPÓTESIS, PREGUNTA, IDEA A DEFENDER. Quito. Ecuador 
Año 13.

Revista de Investigación

Núm. 33

Académica sin Frontera

https://revistainvestigacionacademicasinfrontera.unison.mx/index.php/RDIASF

Recibido el 30 de abril de 2020. Dictaminado mediante arbitraje favorablemente 22 de septiembre de 2020.

8. Fernández, J. (2001). Conceptos fundamentales del materialismo religioso. El Basilisco: Revista de Materialismo Filosófico(30), 33-42. Obtenido de https://dialnet.unirioja.es

9. Hernández, R., Fernández, C., \& Baptista, P. (2006). Metodología de la Investigación (4ta ed.). México: Mc Graw Hill Interamericana. Obtenido de https://seminariodemetodologiadelainvestigacion.files.wordpress.com

10. Hurtado, J. (2013). Investigación Holística. La hipótesis en investigación, ¿Cuándo se formulan? Caracas, Venezuela: Sypal-Quirón. Obtenido de http://investigacionholistica.blogspot.com

11. Kerlinger, F. (1981). Investigación del comportamiento. Técnicas y metodología. México: México: Nueva Editorial Interamericana. Obtenido de https://padron.entretemas.com

12. Lanuez, M., Martínez, M., \& Pérez, V. (2004). El maestro y la investigación educativa en el siglo XXI. La Habana, Cuba.

13. Larousse. (2007). Diccionario Manual de la Lengua Española. S.L.

14. López, J. (2007). Investigación educativa: en preguntas y respuestas. Curso de metodología para el postgrado. Universidad de Ciencias Pedagógicas «Héctor Alfredo Pineda Zaldívar. La Habana, Cuba. Obtenido de https://scielo.sld.cu

15. Mc Guigan, F. (1998). Psicología Experimental. Enfoque metodológico. México: Trillas. Obtenido de https://es.scribd.com

16. Pérez, G., García, G., Nocedo, I., \& García, M. (1996). Metodología de la Investigación Educacional, Primera parte. Ciudad de La Habana, Cuba: Pueblo y Educación. Obtenido de https://www.academia.edu 
"Fl wher ide mis hijos

Año 13.

Núm. 33

\section{httpse//revis}

Revista de Investigación

Académica sin Frontera

ISSN: 2007-8870

Recibido el 30 de abril de 2020. Dictaminado mediante arbitraje favorablemente 22 de septiembre de 2020.

17. Viciedo, C. (1996). Metodología de la Investigación Educativa, Curso internacional de Maestría en Educación Superior. Potosí, Bolivia. Obtenido de http://metodologiaroxana.blogspot.com/p/bibliografia.html 
Año 13.

Revista de Investigación

Núm. 33

Académica sin Frontera

https://revistainvestigacionacademicasinfrontera.unison.mx/index.php/RDIASF

Recibido el 30 de abril de 2020. Dictaminado mediante arbitraje favorablemente 22 de septiembre de 2020.

\section{Comité editorial}

Dra. Angélica María Rascón Larios

Universidad de Sonora. México

Dra. María del Rosario Molina González

Universidad de Sonora

Dra. Francisca Elena Rochin Wong

Universidad de Sonora. México

Dra. Lidia Amalia Zallas Esquer

Universidad de Sonora. México

Dra. Beatriz Llamas Arechiga

Universidad de Sonora. México

Dr. Rogelio Barba Álvarez

Universidad de Guadalajara. México

Dra. Rosa María Rincón Ornelas

Universidad de Sonora. México

Dr. Juan Flores Preciado

Universidad de Colima. México

Dr. Amado Olivares Leal. Universidad de Sonora

Universidad de Sonora. México

Dr. Guillermo Velázquez Valadez.

Instituto Politécnico Nacional (IPN) México

Dr. Hugo Nefstalí Padilla Torres.

Universidad Estatal de Sonora. México

Dr. Luis Ramón Moreno Moreno.

Universidad Autónoma de Baja California. México

Dr. Miguel Ángel Vázquez Ruiz.

Universidad de Sonora. México 
Año 13.

Revista de Investigación

Núm. 33

Académica sin Frontera

ISSN: 2007-8870

https://revistainvestigacionacademicasinfrontera.unison.mx/index.php/RDIASF

Recibido el 30 de abril de 2020. Dictaminado mediante arbitraje favorablemente 22 de septiembre de 2020.

Dra. Lorena Vélez García.

Universidad Autónoma de Baja California. México

Dra. Pabla Peralta Miranda.

Universidad Simón Bolívar, Barranquilla, Colombia

Mtro. Roberto Espíritu Olmos

Universidad de Colima (FCA Tecomán) Colima

Dr. Héctor Priego Huertas.

Universidad de Colima (FCA Tecomán) Colima

Mtra. María Guadalupe Alvarado Ibarra.

Universidad de Sonora. México.

MSc. Celso Germán Sánchez Zayas

Universidad de Camagüey, Ignacio Agramonte Loynaz, Cuba

Dra. María Luisa Quintero Soto

Universidad Autónoma del Estado de México

Dr. Eyder Bolivar Mojica

Universidad Católica, Luis Amigó, Medellin, Colombia

Revisores de Textos en Inglés

Mtro. Renato Encinas

Mtra. Cecilia Guadalupe Martínez Solano

\section{Comité científico}

Dr. Rosendo Martínez Jiménez. Universidad Autónoma Benito Juárez de Oaxaca.

Dr. Hugo Neftalí Padilla. Universidad Estatal de Sonora

Dra. María Teresa Gaxiola Sánchez. Universidad de Sonora.

Dr. José Cesar Kaplan. Universidad Estatal de Sonora.

Dr. Alfredo Islas Rodríguez. Universidad de Sonora

Frecuencia de publicación: semestral / 2 números por año. 


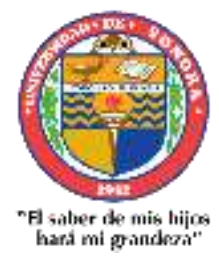

( Julio - Diciembre 2020)

Año 13.

Revista de Investigación

Núm. 33

Académica sin Frontera

ISSN: 2007-8870

https://revistainvestigacionacademicasinfrontera.unison.mx/index.php/RDIASF

Recibido el 30 de abril de 2020. Dictaminado mediante arbitraje favorablemente 22 de septiembre de 2020.

Revista de Investigación Académica sin Frontera (RIASF) con (ISSN: 2007-8870) es un interlocutor internacional de acceso abierto revisado diario en línea en el ámbito del de las Ciencias Económicas Administrativas y Sociales. Su objetivo principal es dar a los trabajos de investigación de calidad. Cubre todas las sub-campos de los campos anteriormente mencionados. Proporciona la plataforma a académicos, estudiantes y profesionales. Sólo pública trabajos de investigación y artículos de revisión inicial. Documento presentado debe cumplir con algunos criterios como, debe ser original, inédita y no estén sometidos a ninguna otra revista.

RIASF es una revista arbitrada / Revisión por pares International. Publicamos documentos sobre una variedad de temas, contextos y estrategias de análisis que examinan la relación entre la rápida evolución para la Sociedad y la tecnología del conocimiento.

REVISTA DE INVESTIGACIÓN ACADÉMICA SIN FRONTERA, Año 13, No. 33, Julio - diciembre 2020, es una publicación semestral de investigación científica, editada por la Universidad de Sonora, a través de las División de Ciencias Económicas y Sociales, de la Unidad Regional Sur, Blvd. Lázaro Cárdenas No. 100, Col. Francisco Villa, Navojoa, Sonora, Sonora, México, C.P. 85880. Tel. (642) 425-99-54.

http://www.revistainvestigacionacademicasinfrontera.com/, revistaacademicasinfrontera@unison.mx.

Editor responsable: Francisco Espinoza Morales. Reserva de Derechos al Uso Exclusivo: 04-2013121811323700-203 e ISSN: 2007-8870, ambos otorgados por el Instituto Nacional de Derecho de Autor. Inscrita en el Directorio de LATINDEX, con Núm. De folio 20014, folio único 14590. Responsable de la última actualización de este Número, Unidad Informática de la Universidad de Sonora, fecha de la última modificación, 30 de diciembre 2020, indexada a Cite Factor Academic Scientific Journal y Journals Master (IIJIF) y Red Latinoamericana de Revistas Académicas en Ciencias Sociales y Humanidades, (Latín Rev). Las opiniones expresadas por los autores no necesariamente reflejan la postura del editor de la publicación. Se autoriza la reproducción total o parcial de los contenidos e imágenes en la presente publicación siempre y cuando se cuente con la autorización del editor y se cite plenamente la fuente. 
Año 13.

Revista de Investigación

Núm. 33

Académica sin Frontera

ISSN: 2007-8870

https://revistainvestigacionacademicasinfrontera.unison.mx/index.php/RDIASF

Recibido el 30 de abril de 2020. Dictaminado mediante arbitraje favorablemente 22 de septiembre de 2020.

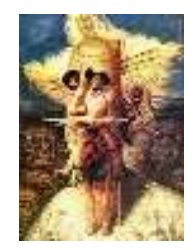

Nos complace anunciar que su diario, "Academic Research Journal Withoutborders" (ISSN/EISSN 20078870) fue evaluado positivamente en la indexación Citefactor, ahora la página de la revista está disponible en línea, en caso de cualquier problema.

Journals Master | International Innovative Journal Impact Factor (IIJIF)

Red Latinoamericana de revistas Académicas en Ciencias Sociales y Humanidades
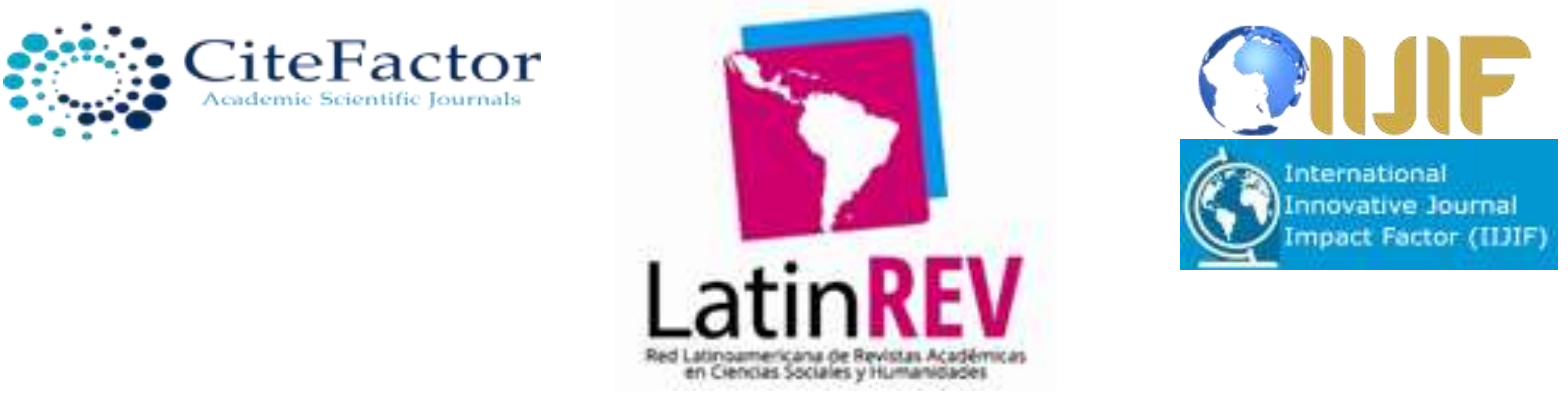

https://www.neliti.com

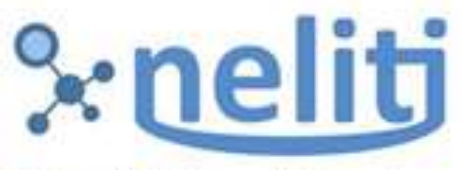

Indonesia's Research Repository
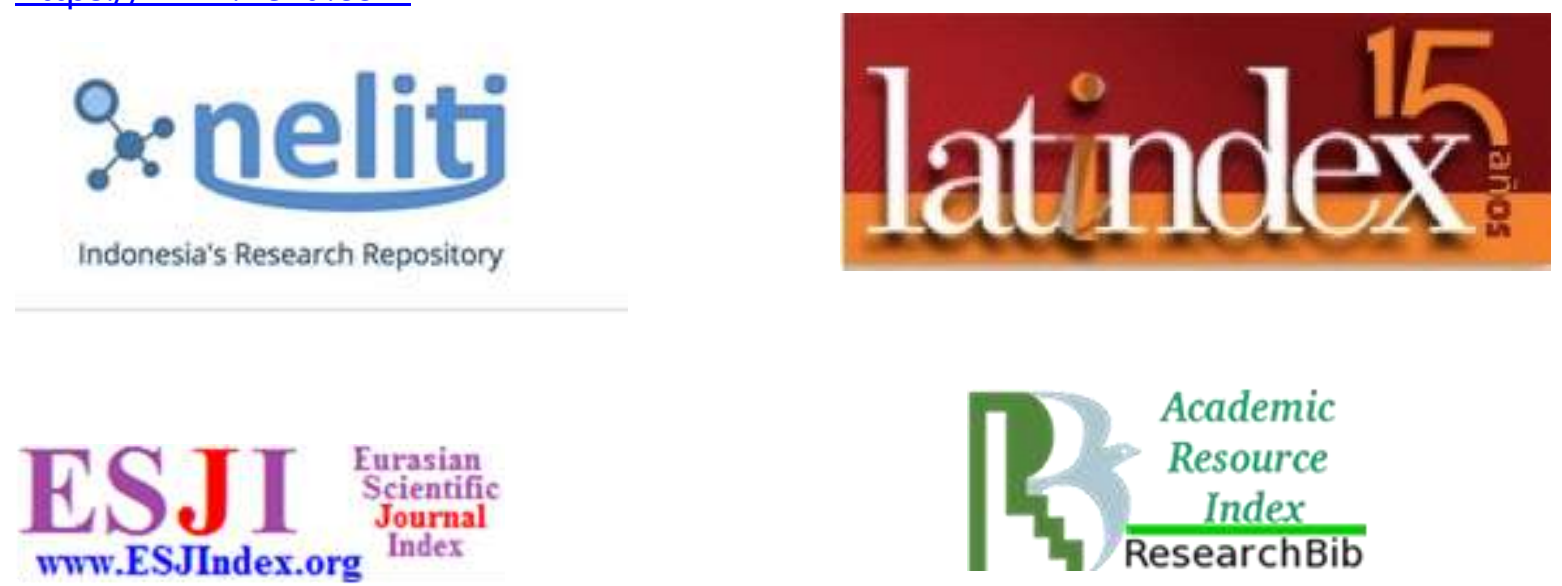
Revista de Investigación

Año 13.

Académica sin Frontera

Núm. 33

ISSN: 2007-8870

https://revistainvestigacionacademicasinfrontera.unison.mx/index.php/RDIASF

Recibido el 30 de abril de 2020. Dictaminado mediante arbitraje favorablemente 22 de septiembre de 2020. 